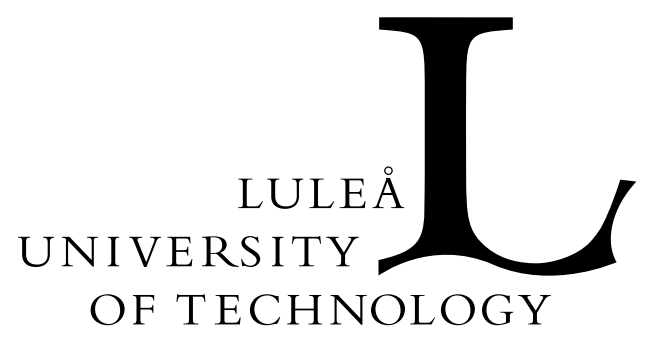

Division of Signal Processing

Visiting address: Universitetsområdet, Porsön, Luleå Postal address: SE-971 87, Luleå, Sweden

Telephone: +46920910 00. Fax: +4692072043

URL: http://www.sm.luth.se/csee/sp/

\title{
On Channel Estimation In OFDM Systems
}

\section{Jan-Jaap van de Beek, Ove Edfors, Magnus Sandell, Sarah Kate Wilson and Per Ola Börjesson}

In Proceedings of Vehicular Technology Conference (VTC '95), vol. 2, pp. 815-819, Chicago, USA, September 1995.

(C) 1995 IEEE. Personal use of this material is permitted. However, permission to reprint/republish this material for advertising or promotional purposes or for creating new collective works for resale or redistribution to servers or lists, or to reuse any copyrighted component of this work in other works must be obtained from the IEEE. 


\title{
On Channel Estimation in OFDM Systems
}

\author{
Jan-Jaap van de Beek* Ove Edfors* Magnus Sandell* \\ Sarah Kate Wilson ${ }^{\dagger} \quad$ Per Ola Börjesson* \\ * Div. of Signal Processing \\ Luleå University of Technology \\ S-971 87 Luleå, Sweden \\ $\dagger$ Purdue University \\ School of Electrical Engineering \\ 1285 Electrical Engineering Bldg \\ West Lafayette, IN 47907-1285, USA
}

\begin{abstract}
The use of multi-amplitude signaling schemes in wireless OFDM systems requires the tracking of the fading radio channel. This paper addresses channel estimation based on time-domain channel statistics. Using a general model for a slowly fading channel, we present the MMSE and LS estimators and a method for modifications compromising between complexity and performance. The symbol error rate for a 16-QAM system is presented by means of simulation results. Depending upon estimator complexity, up to $4 \mathrm{~dB}$ in SNR can be gained over the LS estimator.
\end{abstract}

\section{INTRODUCTION}

Currently, orthogonal frequency-division multiplexing (OFDM) systems [1] are subject to significant investigation. Since this technique has been adopted in the European digital audio broadcasting (DAB) system [2], OFDM signaling in fading channel environments has gained a broad interest. For instance, its applicability to digital TV broadcasting is currently being investigated [3].

The use of differential phase-shift keying (DPSK) in OFDM systems avoids the tracking of a time varying channel. However, this will limit the number of bits per symbol and results in a $3 \mathrm{~dB}$ loss in signal-to-noise ratio (SNR) [4]. If the receiver contains a channel estimator, multiamplitude signaling schemes can be used.

In [5] and [6], 16-QAM modulation in an OFDM system has been investigated. A decision-directed channeltracking method, which allows the use of multi-amplitude schemes in a slow Rayleigh-fading environment is analysed in [5].

In the design of wireless OFDM systems, the channel is usually assumed to have a finite-length impulse response. A cyclic extension, longer than this impulse response, is put between consecutive blocks in order to avoid interblock interference and preserve orthogonality of the tones [7]. Generally, the OFDM system is designed so that the cyclic extension is a small percentage of the total symbol length. This paper discusses channel estimation techniques in wireless OFDM systems, that use this property of the channel impulse response. Hoeher [6] and Cioffi [8] have also addressed this property.

In Section II, we describe the system model. Section III discusses the minimum mean-square error (MMSE) and least-squares (LS) channel estimators. The MMSE estimator has good performance but high complexity. The LS estimator has low complexity, but its performance is not as good as that of the MMSE estimator. We present modifications to both MMSE and LS estimators that use the assumption of a finite length impulse response. In Section IV we evaluate the estimators by simulating a 16-QAM signaling scheme. The performance is presented both in terms of mean-square error (MSE) and symbol error rate (SER).

\section{System Description}

We will consider the system shown in Fig. 1 , where $x_{k}$ are the transmitted symbols, $g(t)$ is the channel impulse response, $\widetilde{n}(t)$ is the white complex Gaussian channel noise and $y_{k}$ are the received symbols. The transmitted symbols $x_{k}$ are taken from a multi-amplitude signal constellation. The D/A and A/D converters contain ideal low-pass filters with bandwidth $1 / T_{s}$, where $T_{s}$ is the sampling interval. A cyclic extension of time length $T_{G}$ (not shown in Fig. 1 for reasons of simplicity) is used to eliminate inter-block interference and preserve the orthogonality of the tones.

We treat the channel impulse response $g(t)$ as a timelimited pulse train of the form

$$
g(t)=\sum_{m} \alpha_{m} \delta\left(t-\tau_{m} T_{s}\right)
$$

where the amplitudes $\alpha_{m}$ are complex valued and $0 \leq$ $\tau_{m} T_{s} \leq T_{G}$, i.e., the entire impulse response lies inside

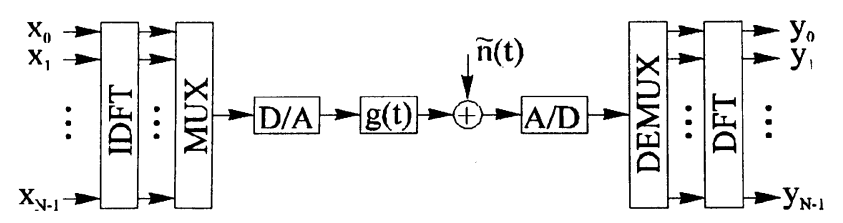

Fig. 1: Base-band OFDM system. 


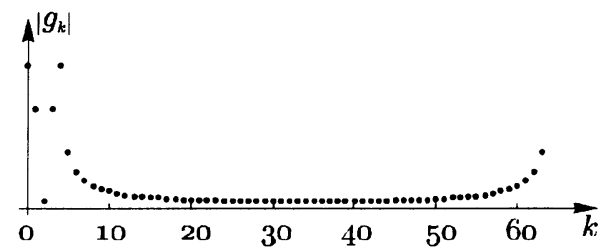

Fig. 2: Leakage between taps for the continuous channel $g(t)=\delta\left(t-0.5 T_{s}\right)+\delta\left(t-3.5 T_{s}\right)$.

the guard space. The system is then modelled using the $N$-point discrete-time Fourier transform $\left(\mathrm{DFT}_{\mathrm{N}}\right)$ as

$$
\mathbf{y}=\operatorname{DFT}_{\mathrm{N}}\left(\operatorname{IDFT}_{\mathrm{N}}(\mathbf{x}) \circledast \frac{\mathbf{g}}{\sqrt{\mathbf{N}}}+\tilde{\mathbf{n}}\right)
$$

where $\circledast$ denotes cyclic convolution, $\mathbf{x}=\left[\begin{array}{lll}x_{0} & x_{1} \ldots x_{N-1}\end{array}\right]^{T}$, $\mathbf{y}=\left[\begin{array}{llll}y_{0} & y_{1} \ldots y_{N-1}\end{array}\right]^{T}, \widetilde{\mathbf{n}}=\left[\begin{array}{llll}\widetilde{n}_{0} & \widetilde{n}_{1} \ldots \widetilde{n}_{N-1}\end{array}\right]^{T}$ is a vector of i.i.d. complex Gaussian variables, and $\mathbf{g}=\left[\begin{array}{lll}g_{0} & g_{1} \ldots g_{N-1}\end{array}\right]^{T}$ is determined by the cyclic equivalent of sinc-functions. The vector $\mathrm{g} / \sqrt{N}$ is the observed channel impulse response after sampling the frequency response of $g(t)$, and

$$
g_{k}=\frac{1}{\sqrt{N}} \sum_{m} \alpha_{m} e^{-j \frac{\pi}{N}\left(k+(N-1) \tau_{m}\right)} \frac{\sin \left(\pi \tau_{m}\right)}{\sin \left(\frac{\pi}{N}\left(\tau_{m}-k\right)\right)} .
$$

The validity of the cyclic model described by (2) and (3) depends on how well the objective of the guardspace is met, i.e., how well it eliminates inter-block interference.

If the delay $\tau_{m}$ is an integer, then all the energy from $\alpha_{m}$ is mapped to tap $g_{\tau_{m}}$. However, for a non- $T$-spaced pulse, i.e., if $\tau_{m}$ is not an integer, its energy will leak to all taps $g_{k}$. Fig. 2 illustrates this leakage for a special case. Notice that most of the energy is kept in the neighbourhood of the original pulse locations.

The system described by (2) can be written as a set of $N$ independent Gaussian channels, see Fig. 3,

$$
y_{k}=h_{k} x_{k}+n_{k}, \quad k=0 \ldots N-1,
$$

where $h_{k}$ is the complex channel attenuation given by $\mathbf{h}=$ $\left[h_{0} h_{1} \ldots h_{N-1}\right]^{T}=\mathrm{DFT}_{\mathrm{N}}(\mathrm{g})$ and $\mathbf{n}=\left[\begin{array}{ll}n_{0} & n_{1} \ldots n_{N-1}\end{array}\right]^{T}=$ $\operatorname{DFT}_{N}(\widetilde{\mathbf{n}})$ is an i.i.d. complex zero-mean Gaussian noise vector.

As a matter of convenience, we write (4) in matrix notation

$$
\mathbf{y}=\mathbf{X F g}+\mathbf{n},
$$

where $\mathbf{X}$ is a matrix with the elements of $\mathbf{x}$ on its diagonal and

$$
\mathbf{F}=\left[\begin{array}{ccc}
W_{N}^{00} & \cdots & W_{N}^{0(N-1)} \\
\vdots & \ddots & \vdots \\
W_{N}^{(N-1) 0} & \cdots & W_{N}^{(N-1)(N-1)}
\end{array}\right]
$$

is the DFT-matrix with

$$
W_{N}^{n k}=\frac{1}{\sqrt{N}} e^{-j 2 \pi \frac{n k}{N}} .
$$

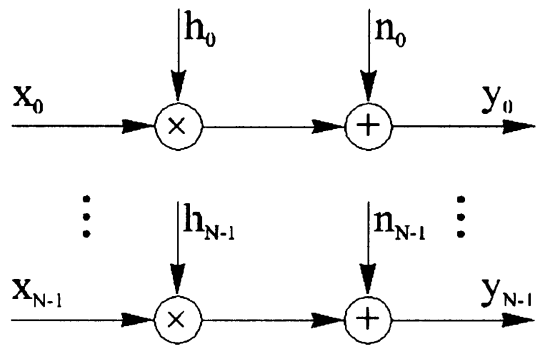

Fig. 3: Parallel Gaussian channels.

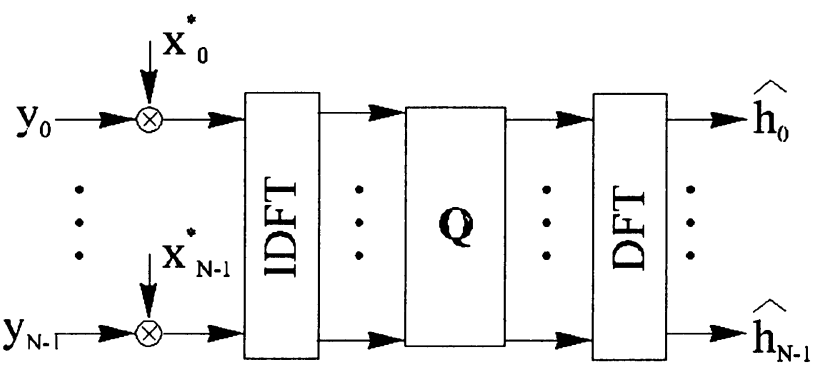

Fig. 4: General estimator structure.

\section{Channel Estimation}

We will derive several estimators based on the system model in the previous section. These estimation techniques all have the general structure presented in Fig. 4. The transmitted symbols $x_{k}$, appearing in the estimator expressions, are either training symbols or quantized decision variables in a decision-directed estimator. Error propagation in the decision-directed case is not treated in this paper.

\section{A. MMSE and LS Estimators}

If the channel vector $\mathbf{g}$ is Gaussian and uncorrelated with the channel noise $\mathbf{n}$, the MMSE estimate of $\mathbf{g}$ becomes [9]

$$
\widehat{\mathbf{g}}_{\mathrm{MMSE}}=\mathbf{R}_{\mathbf{g y}} \mathbf{R}_{\mathbf{y y}}^{-1} \mathbf{y}
$$

where

$$
\begin{aligned}
& \mathbf{R}_{\mathbf{g y}}=\mathrm{E}\left\{\mathbf{g y}^{H}\right\}=\mathbf{R}_{\mathbf{g g}} \mathbf{F}^{H} \mathbf{X}^{H} \\
& \mathbf{R}_{\mathbf{y y}}=\mathrm{E}\left\{\mathbf{y} \mathbf{y}^{H}\right\}=\mathbf{X F R}_{\mathbf{g g}} \mathbf{F}^{H} \mathbf{X}^{H}+\sigma_{n}^{2} \mathbf{I}_{N}
\end{aligned}
$$

are the cross covariance matrix between $\mathbf{g}$ and $\mathbf{y}$ and the auto-covariance matrix of $\mathbf{y}$. Further, $\mathbf{R}_{\mathbf{g g}}$ is the autocovariance matrix of $\mathbf{g}$ and $\sigma_{n}^{2}$ denotes the noise variance $\mathrm{E}\left\{\left|n_{k}\right|^{2}\right\}$. These two quantities are assumed to be known. Since the columns in $\mathbf{F}$ are orthonormal, $\widehat{\mathbf{g}}_{\text {MMSE }}$ generates the frequency-domain MMSE estimate $\widehat{\mathbf{h}}_{\mathrm{MMSE}}$ by

$$
\widehat{\mathbf{h}}_{\mathrm{MMSE}}=\mathbf{F} \widehat{\mathbf{g}}_{\mathrm{MMSE}}=\mathbf{F} \mathbf{Q}_{\mathrm{MMSE}} \mathbf{F}^{H} \mathbf{X}^{H} \mathbf{y},
$$


where $\mathbf{Q}_{\mathrm{MMSE}}$ can be shown to be

$$
\mathbf{Q}_{\mathrm{MMSE}}=\mathbf{R}_{\mathbf{g g}}\left[\left(\mathbf{F}^{H} \mathbf{X}^{H} \mathbf{X F}\right)^{-1} \sigma_{n}^{2}+\mathbf{R}_{\mathbf{g g}}\right]^{-1}\left(\mathbf{F}^{H} \mathbf{X}^{H} \mathbf{X F}\right)^{-1} .
$$

This MMSE channel estimator (9) has the form shown in Fig. 4. If $\mathbf{g}$ is not Gaussian, $\widehat{\mathbf{h}}_{\mathrm{MMSE}}$ is not necessarily a minimum mean-square error estimator. It is however the best linear estimator in the mean-square error sense. In either case (g, Gaussian or not) we will denote the channel estimate as $\widehat{\mathbf{h}}_{\mathrm{MMSE}}$.

The LS estimator for the cyclic impulse response $\mathrm{g}$ minimizes $(\mathbf{y}-\mathbf{X F g})^{H}(\mathbf{y}-\mathbf{X F g})$ and generates

$$
\widehat{\mathbf{h}}_{\mathrm{LS}}=\mathbf{F Q}_{\mathrm{LS}} \mathbf{F}^{H} \mathbf{X}^{H} \mathbf{y}
$$

where

$$
\mathbf{Q}_{\mathrm{LS}}=\left(\mathbf{F}^{H} \mathbf{X}^{H} \mathbf{X F}\right)^{-1}
$$

Note that $\widehat{\mathbf{h}}_{\mathrm{LS}}$ also corresponds to the estimator structure in Fig. 4. Since (11) reduces to

$$
\widehat{\mathbf{h}}_{\mathrm{LS}}=\mathbf{X}^{-1} \mathbf{y}
$$

the LS estimator is equivalent to what is also referred to as the zero-forcing estimator.

Both estimators (9) and (13) have their drawbacks. The MMSE estimator suffers from a high complexity, whereas the LS estimate has a high mean-square error. In the next section, we will address these drawbacks and modify both estimators.

\section{B. Modified MMSE and LS Estimators}

The MMSE estimator requires the calculation of an $N \times N$ matrix $\mathbf{Q}_{\mathrm{MMSE}}$, which implies a high complexity when $N$ is large. A straightforward way of decreasing the complexity is to reduce the size of $\mathbf{Q}_{\mathrm{MMSE}}$. As indicated in Fig. 2, most of the energy in $\mathbf{g}$ is contained in, or near, the first $L=\left\lceil\frac{T_{G}}{T_{s}}\right\rceil$ taps. Therefore we study a modification of the MMSE estimator, where only the taps with significant energy are considered. The elements in $\mathbf{R}_{\mathbf{g g}}$ corresponding to low energy taps in $\mathbf{g}$ are approximated by zero.

If we take into account the first $L$ taps of $\mathbf{g}$, and set $\mathbf{R}_{\mathbf{g g}}(r, s)=0$ for $r, s \notin[0, L-1]$, then $\mathbf{Q}_{\mathrm{MMSE}}$ is effectively reduced to an $L \times L$ matrix. If the matrix $\mathbf{T}$ denotes the first $L$ columns of the DFT-matrix $\mathbf{F}$ and $\mathbf{R}_{\mathbf{g g}}^{\prime}$ denotes the upper left $L \times L$ corner of $\mathbf{R}_{\mathbf{g g}}$, the modified MMSE estimator becomes

$$
\widehat{\mathbf{h}}_{\mathrm{MMSE}}=\mathbf{T} \mathbf{Q}_{\mathrm{MMSE}}^{\prime} \mathbf{T}^{H} \mathbf{X}^{H} \mathbf{y}
$$

where

$$
\mathbf{Q}_{\mathrm{MMSE}}^{\prime}=\mathbf{R}_{\mathbf{g g}}^{\prime}\left[\left(\mathbf{T}^{H} \mathbf{X}^{H} \mathbf{X} \mathbf{T}\right)^{-1} \sigma_{n}^{2}+\mathbf{R}_{\mathbf{g g}}^{\prime}\right]^{-1}\left(\mathbf{T}^{H} \mathbf{X}^{H} \mathbf{X} \mathbf{T}\right)^{-1}
$$

This modification is illustrated in Fig. 5. As mentioned in Section I, an OFDM system is usually designed so that

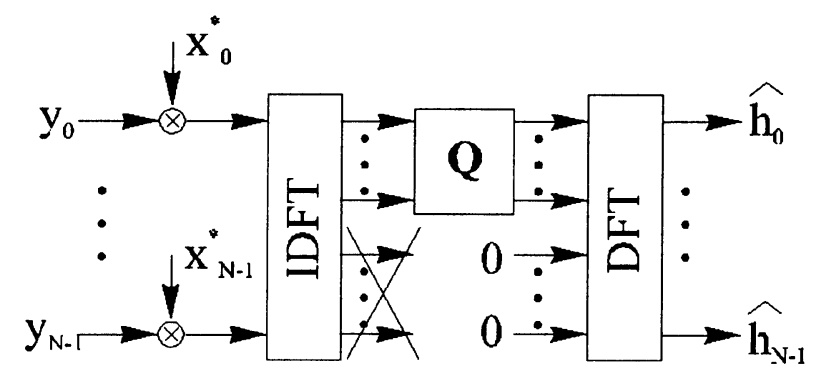

Fig. 5: Modified estimator structure.

$L$ is a small fraction of $N$. Thus, the complexity of the MMSE estimator will decrease considerably.

Although the complexity of the LS estimator does not prompt for modifications, its perfor..1ance in terms of mean-square error can be improved for a range of SNRs by following the same general concept as above. The LS estimator does not use the statistics of the channel. Intuitively, excluding low energy taps of $\mathbf{g}$ will to some extent compensate for this shortcoming since the energy of $\mathbf{g}$ decreases rapidly outside the first $L$ taps, whilst the noise energy is assumed to be constant over the entire range $[6,8]$.

Taking only the first $L$ taps of $\mathbf{g}$ into account, thus implicitly using channel statistics, the modified LS estimator becomes

$$
\widehat{\mathbf{h}}_{\mathrm{LS}}=\mathbf{T} \mathbf{Q}_{\mathrm{LS}}^{\prime} \mathbf{T}^{H} \mathbf{X}^{H} \mathbf{y}
$$

where

$$
\mathbf{Q}_{\mathbf{L S}}^{\prime}=\left(\mathbf{T}^{H} \mathbf{X}^{H} \mathbf{X T}\right)^{-1}
$$

The modified LS estimator also has the structure as shown in Fig. 5.

\section{Estimator Complexity}

The complexity of the modified LS estimator (16) will be larger than that of the full LS estimator, since a simplification as in (13) cannot be performed. Notice that while the full LS estimator (13) has much lower complexity than the full MMSE estimator (9), the respective modified versions (16) and (14) are equally complex.

It should be noted that the MMSE estimators have been derived under the assumption of known channel correlation and noise variance. In practice these quantities, $\mathbf{R}_{\mathbf{g g}}$ and $\sigma_{n}^{2}$, are either taken fixed or estimated, possibly in an adaptive way. This will increase the estimator complexity and reduce the performance slightly.

In the special case where the channel (1) is $T$-spaced, i.e., where $\tau_{m}$ are integers, no leakage of energy to taps outside the interval $[0, L]$ will occur and the two modified estimators (14) and (16) will not lose any information about the channel. Thus, the modified MMSE estimator (14) is equivalent to the MMSE estimator (9). The modified LS estimator (16) in this case will outperform the 
LS estimator (13) for all SNRs, since the excluded taps contain only noise.

More generally, for non- $T$-spaced channels, any subset of the taps in $\mathbf{g}$ may be taken into account when modifying the MMSE and the LS estimator. The size of this subset determines the complexity for both types of modified estimators.

In Section IV we consider the case where this subset consists of the taps $g_{0} \ldots g_{L+K-1}$ and $g_{N-K} \ldots g_{N-1}$, i.e., the first $L$ taps as well as $K$ extra taps on each side.

\section{Simulations}

\section{A. Simulated Channels}

In the simulations we consider a system operating with a bandwidth of $500 \mathrm{kHz}$, divided into 64 tones with a total symbol period of $138 \mu \mathrm{s}$, of which $10 \mu \mathrm{s}$ is a cyclic prefix. Sampling is performed with a $500 \mathrm{kHz}$ rate. A symbol thus consists of 69 samples, five of which are contained in the cyclic prefix (i.e. $L=5$ ). 50,000 channels are randomized per average SNR, each consisting of five pulses, of which four have uniformly distributed delays over the interval 0-10 $\mu \mathrm{s}$, while one tap is always assumed to have a zero delay, corresponding to a perfect time synchronisation of the sampling instants. The multipath intensity profile is assumed to be $\phi(\tau) \sim e^{-\tau / \tau_{\text {rms }}}$, where $\tau_{\text {rms }}$ is $1 / 4$ of the cyclic extension. We have used Monte-Carlo simulations to generate the $\mathbf{R}_{\mathbf{g g}}$ for this channel model. This covariance matrix together with the true noise variance $\sigma_{n}^{2}$ is used in the MMSE estimations to follow. The average SNR per symbol in Fig. 3 is defined as $\mathrm{E}\left\{\left|h_{k}\right|^{2}\right\} / \mathrm{E}\left\{\left|n_{k}\right|^{2}\right\}$, since $\mathrm{E}\left\{\left|x_{k}\right|^{2}\right\}$ is normalised to unity.

The following estimators are used:

\begin{tabular}{|l|l|l|c|}
\hline Estimator & Notation & Taps used & Size $\mathbf{Q}^{\prime}$ \\
\hline \hline MMSE & MMSE & $0 \ldots 63$ & $64 \times 64$ \\
\hline LS & LS & $0 \ldots 63$ & N.A. \\
\hline \multirow{3}{*}{$\begin{array}{l}\text { Modified } \\
\text { MSE }\end{array}$} & MMSE-0 & $0 \ldots 4$ & $5 \times 5$ \\
\cline { 2 - 4 } & MMSE-5 & $0 \ldots 9,59 \ldots 63$ & $15 \times 15$ \\
\cline { 2 - 4 } & MMSE-10 & $0 \ldots 14,54 . .63$ & $25 \times 25$ \\
\hline \multirow{2}{*}{ LS } & LS-0 & $0 \ldots 4$ & $5 \times 5$ \\
\cline { 2 - 4 } & LS-5 & $0 \ldots 9,59 \ldots 63$ & $15 \times 15$ \\
\cline { 2 - 4 } & LS-10 & $0 \ldots 14,54 . .63$ & $25 \times 25$ \\
\hline
\end{tabular}

\section{B. Mean-square Error}

Fig. 6 shows the mean-square error versus SNR for the MMSE, LS, MMSE-0, MMSE-5 and MMSE-10 estimators. The difference between the modified MMSE estimators and the MMSE estimator is due to the fact that parts of the channel statistics are not taken into account in the former. For low SNRs, this approximation effect is small compared to the channel noise, while it becomes dominant for large SNRs. The curves level out to a value determined by the energy in the excluded taps. Larger

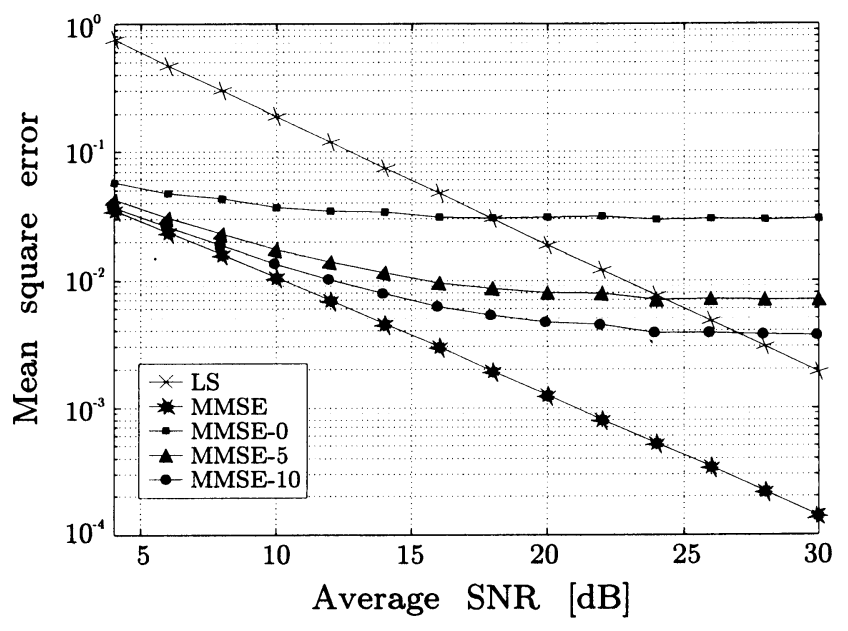

Fig. 6: Mean-square error for three modified MMSE estimators.

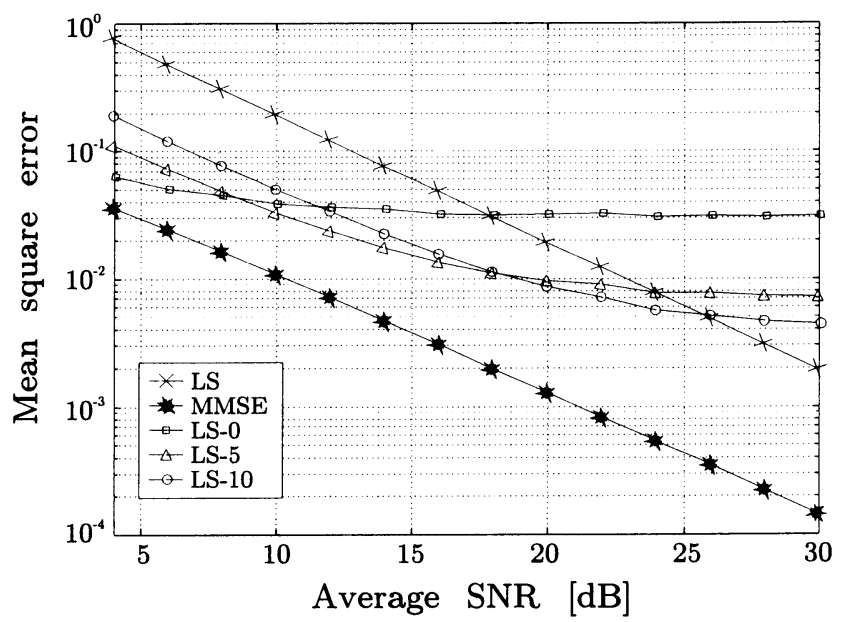

Fig. 7: Mean-square error for three LS estimators.

dimensions of $\mathbf{Q}_{M}^{\prime}$ will give lower mean-square error for all SNRs.

Fig. 7 shows the mean-square error versus average SNR for the MMSE, LS, LS-0, LS-5 and LS-10 estimators. Contrary to the modification of the MMSE estimator, the modification of the LS estimator reduces the mean-square error for a range of SNRs. However, the same approximation effect as in the modified MMSE estimators shows up at high SNRs. An interesting observation is, that for every SNR there exists an optimal size of $\mathbf{Q}_{\mathrm{LS}}^{\prime}$, which gives the smallest mean-square error compared to the other modified LS estimators.

\section{Symbol-error Rate}

The symbol-error rate (SER) curves presented in this section are based on the mean-square errors of the channel estimations presented in the previous section. For the calculation of SER, we have used the formulae presented in [10]. These formulae find the symbol error rate of a 


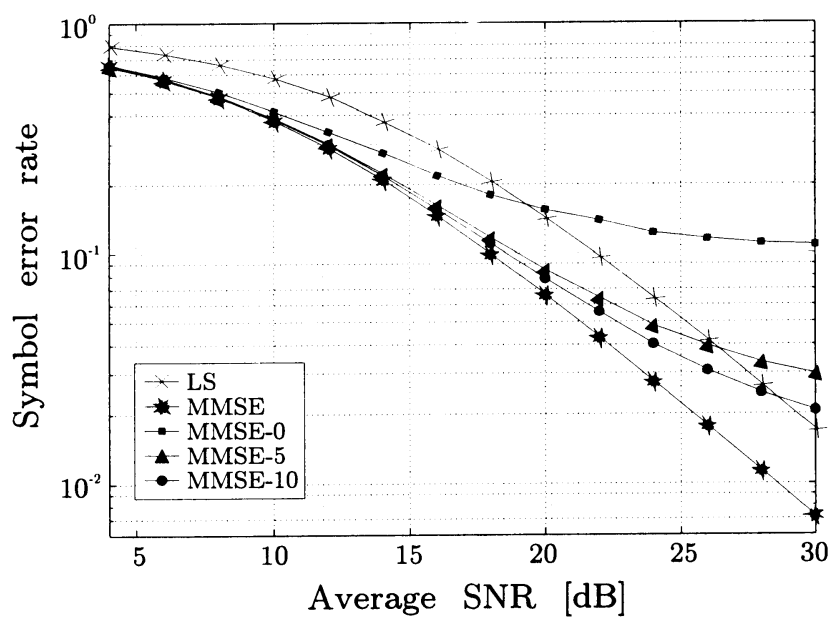

Fig. 8: Symbol error rate for three modified MMSE estimators.

16-QAM system given a noisy estimate of the channel. We consider decision-directed estimation, without error propagation.

As seen in Fig. 8, a gain in SNR up to $4 \mathrm{~dB}$ can be obtained for certain SNRs when using a modified MMSE estimator instead of the LS estimator, depending on admissible complexity. The same behaviour can be observed for modified LS estimators in Fig. 9. However, the gain in SNR is not as large as for the modified MMSE estimators with the same size of the matrix $\mathbf{Q}$. This is explicitly shown for MMSE-10 and LS-10 in Fig. 10. The difference in SNR between these two estimators is about $2 \mathrm{~dB}$.

\section{CONClusions}

The estimators in this study can be used to efficiently estimate the channel in an OFDM system given a certain knowledge about the channel statistics. The MMSE estimator assumes a priori knowledge of noise variance and channel covariance. Moreover, its complexity is large compared to the LS estimator. For high SNRs the LS estimator is both simple and adequate. However, for low SNRs, the presented modifications of the MMSE and LS estimators will allow a compromise between estimator complexity and performance. For a 16-QAM signaling constellation, up to $4 \mathrm{~dB}$ gain in SNR over the LS estimator was obtained, depending on estimator complexity. Even relatively low-complex modified estimators, however, perform significantly better than the LS estimator for a range of SNRs.

\section{REFERENCES}

[1] Leonard J. Cimini, Jr., "Analysis and simulation of a digital mobile channel using orthogonal frequency-division multiplexing", IEEE Trans. Comm., Vol. 33, no. 7, pp 665-675, July 1985 .

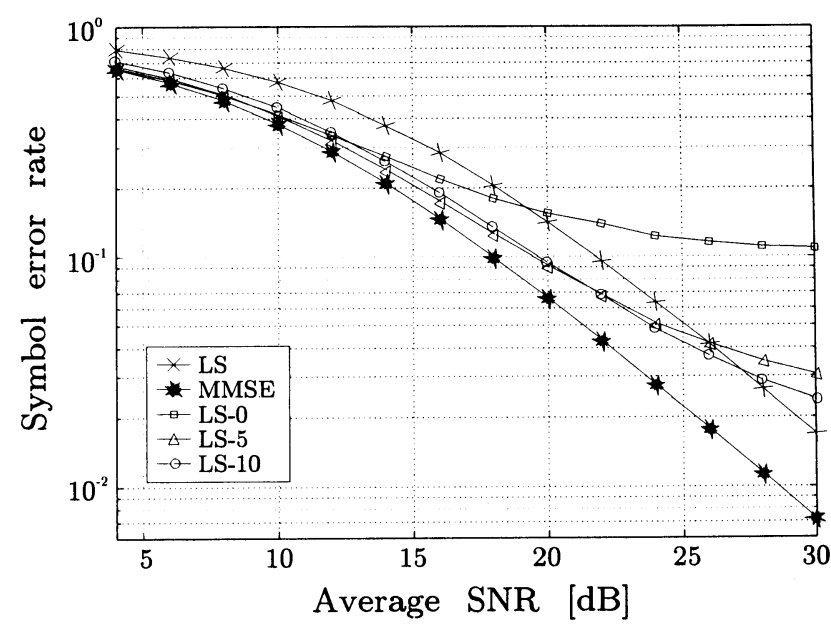

Fig. 9: Symbol error rate for three modifitd LS estimators.

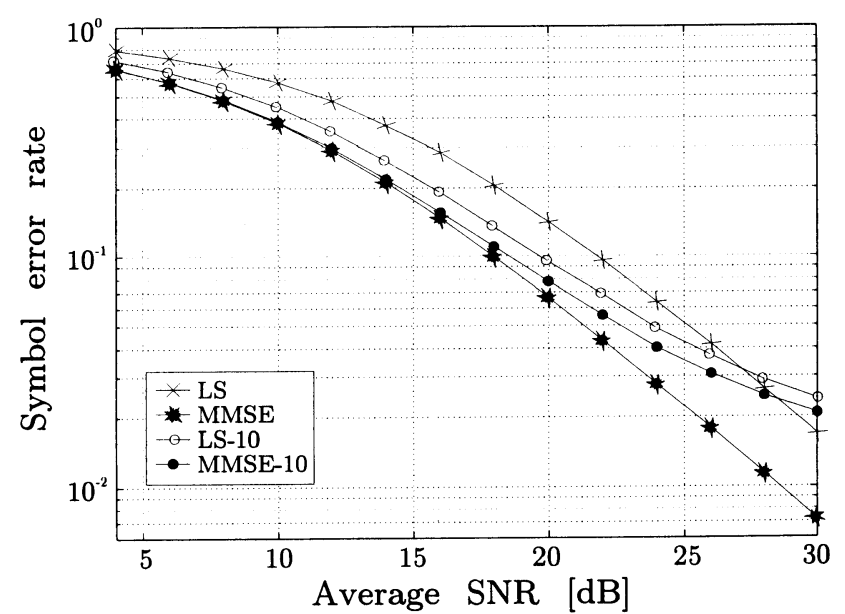

Fig. 10: Comparison of SER between a modified MMSE estimator and the corresonding modified LS estimator.

[2] M. Alard and R. Lassalle, "Principles of modulation and channel coding for digital broadcasting for mobile receivers", $E B U$ Review, no. 224, pp 3-25, August 1987.

[3] B. Marti et al., "European activities on digital television broadcasting-from company to cooperative projects", EBU Technical Review, no. 256, pp. 20-29, 1993.

[4] John Proakis, Digital Communications, McGraw-Hill, 1989.

[5] Sarah Kate Wilson, R. Ellen Khayata and John M. Cioffi, "16-QAM modulation with orthogonal frequency-division multiplexing in a Rayleigh-fading environment", In Proc. VTC1994, pp. 1660-1664, Stockholm, Sweden, June 1994.

[6] Peter Hoeher, "TCM on frequency-selective land-mobile fading channels", In Proc. of the 5th Tirrenia International Workshop on Digital Communications, Tirrenia, Italy, September 1991.

[7] John A.C. Bingham, "Multicarrier modulation for data transmission: an idea whose time has come", IEEE Communications Magazine, 28(5):5-14, May 1990

[8] John M. Cioffi, Stanford University, Private communication.

[9] Louis L. Scharf, Statistical Signal Processing, Addison-Wesley, 1991.

[10] Sarah Kate Wilson, Digital Audio Broadcasting in a Fading and Dispersive Channel, PhD-thesis, Stanford University, August 1994. 